\title{
Control of Retinal and Choroidal Blood Flow
}

\author{
A. BILL and G. O. SPERBER \\ Uppsala, Sweden
}

\begin{abstract}
Summary
Earlier studies on the control of retinal and choroidal blood flow are reviewed and some recent observations on the effects of light on retinal metabolism and retinal and choroidal blood flow in monkeys (Macaca fascicularis) are reported in preliminary form. The retina is nourished by the retinal blood vessels, where blood flow is autoregulated and the choroidal blood vessels where autoregulation is absent. Studies with the deoxyglucose method of Sokoloff indicate that flickering light tends to increase the metabolism of the inner retina, while constant light reduces the metabolism in the outer retina. Retinal blood flow in flickering light, $8 \mathrm{~Hz}$, is higher than in constant light. The sympathetic nerves of the choroid are probably involved in a protective mechanism, preventing overperfusion in fight and flight situations with acute increments in blood pressure. The facial nerve contains parasympathetic vasodilator fibres to the choroid; the physiological significance of these fibres is unknown. The neuropeptides NPY, VIP and PHI are likely to be involved in autonomic reflexes in the eye.
\end{abstract}

\section{Choroidal and retinal blood flow}

In most tissues, the control of the circulation is complex because of the many factors influencing the vascular resistance: local myogenic responses, endothelium derived substances and local metabolic factors as well as circulating substances and the autonomic nerves. The general strategy is that local factors strive to optimise the conditions with regard to such factors as carbon dioxide and oxygen tensions and $\mathrm{pH}$, (autoregulation of blood flow), while circulating factors and autonomic nerves are involved in moment to moment adjustment of the distribution of the cardiac output according to certain priorities. There are great variations between tissues with respect to the relative importance of local and other factors.

In this communication we will give an overview of earlier work concerning the control of choroidal and retinal circulation mainly via autonomic nerves and report on some recent observations on effects of light on retinal metabolism and blood flow.

The blood flow, Q, in the intraocular tissues as in other tissues depends on the local arterial blood pressure, $\mathrm{P}_{\mathrm{a}}$, the local venous pressure and the resistance to flow, $R$. In the eye the pertinent venous pressure for practical purposes can be assumed to equal the intraocular pressure, IOP, except at very low intraocular pressures. ${ }^{1}$ The perfusion pressure for blood flow through the eye thus equals the local arterial blood pressure in the arteries entering the intraocular tissues minus the IOP. The two pressures have to be measured with the same zero level, for obvious reasons.

$$
\mathrm{Q}=\left(\mathrm{P}_{\mathrm{a}}-\mathrm{IOP}\right) / \mathrm{R}
$$

A reduction in perfusion pressure may be From Department of Physiology and Medical Biophysics, University of Uppsala, Sweden.

Correspondence to: Professor A. Bill, Department of Physiology and Medical Biophysics, BMC, Box 572, S-751 23 Uppsala, Sweden. 
caused by a rise in IOP as well as a fall in systemic blood pressure, but it may also be caused by changing the position of the body. Thus in the sitting or standing positions the perfusion pressure is lower than in the recumbent position, due to the decrease in local arterial blood pressure that takes place for hydrostatic reasons. This is not compensated by the slight decrease in IOP that may take place.

Studies in different species have demonstrated that reductions in perfusion pressure cause corresponding reductions in choroidal blood flow. In the retina on the other hand moderate changes in perfusion pressure have only minor or no effects on the blood flow in experimental animals ${ }^{1}$ as well as man. ${ }^{2}$ Alterations in perfusion pressure are thus compensated by factors that strive to maintain normal blood flow in the retina but not in the choroid.

The difference in control between the retinal and choroidal vessels may be explained by the location of the vessels and differences in blood flow rates; in the retina the blood vessels are distributed within the inner layers of the tissue and the metabolic situation in the tissue may thus affect the resistance vessels directly. The oxygen extraction is about $40-50 \%,{ }^{3}$ which is similar to that in the brain. The extraretinal supply to the retina from the choroid is an obvious optical advantage but leads to diffusion over relatively large distances which requires high extravascular concentrations of substrates in the choroid. The oxygen tension as well as the glucose concentration in the tissue fluid of the choroid are indeed exceptionally high, little lower than in arterial blood. ${ }^{4}$

There is a steep fall in oxygen tension in the outer retina. ${ }^{5}$ The minimum is located in the region of the outer nuclear layer. ${ }^{6}$ Thus in this region the oxygen consumed comes both from the retinal and choroidal vascular beds. A prerequisite for the high extravascular concentrations of substrates in the choroid is that blood flow per gram tissue is high; it is in fact higher than in any other tissue of the body. ${ }^{1}$ The arrangement of the blood vessels of the choroid is another interesting feature. The arteries and arterioles are separated from the retina by the choriocapillaris layer. With this arrangement there can be little influence by metabolites from the retina on the choroidal vascular resistance.

It seems likely that myogenic factors and local oxygen and carbon dioxide tensions and $\mathrm{pH}$ as well as metabolites resulting from any tendency to abnormal metabolism are involved in autoregulation of retinal blood flow. There is also the possibility that locally released transmitters involved in vision may affect the retinal blood vessels in a way that cannot be foreseen from experiments with intravascular administration; the blood-retinal barrier may prevent diffusion of the transmitter from blood into the retina.

In most tissues there is a need for variations in nutrition based on variations in activity. In vitro studies have indicated that there may be variations in activity also in the retina. It does then become an intriguing question whether variations in retinal activity are accompanied by variations in retinal and choroidal blood flow. Retinal blood flow might change due to autoregulatory mechanisms, but for variations in choroidal blood flow to occur there would probably be a need for the involvement of autonomic nerves.

\section{Role of autonomic nerves}

The eye has a rich supply of autonomitic nerves, but these nerves are distributed only within the uvea and the extraocular parts of the retinal blood vessels. Sympathetic nerves originate in the superior cervical sympathetic ganglion, parasympathetic nerves in the pterygopalatine and ciliary ganglia. Experiments with electrical stimulation of the sympathetic and facial and oculomotor nerves have revealed effects of such stimulations but the precise role of the nerves is not very well understood.

Choroidal vasodilatation achieved by intracranial stimulation of the oculomotor nerve is moderate, long-lasting and may in fact be due to simultaneous antidromic co-stimulation of the ophthalmic division of the trigeminal nerve. ${ }^{7}$ It will not be discussed any further here.

Stimulation of the cervical sympathetic chain causes frequency dependent vasoconstriction and reduced blood flow in the choroid with near maximal effect at $10 \mathrm{~Hz}$. ${ }^{1}$ There is practically no effect on retinal blood flow 


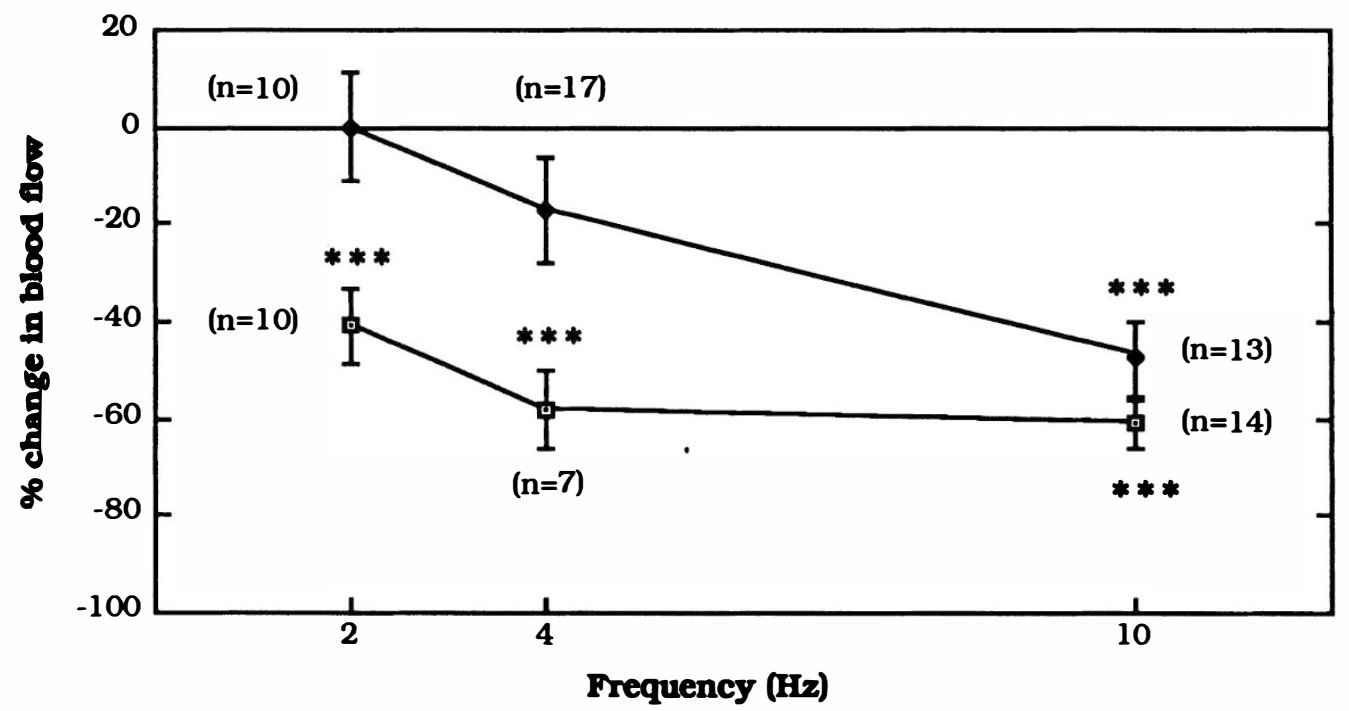

Fig. 1. The effect of sympathetic nerve stimulation at different frequencies on choriodal blood flow under normal conditions $\square$ and after blockade of the alpha-adrenergic receptors $>$. The blood flow was determined with the labelled microsphere technique. From Granstam and Nilsson?.

and also the preretinal oxygen tension is essentially unaffected even at high frequencies indicating that the oxygen tension in the inner parts of the retina is well maintained.

In conscious rabbits there is practically no spontaneous tone in the sympathetic nerves to the uvea, and the same is true under pentobarbital anaesthesia. However under urethan anaesthesia there is a tone corresponding to a stimulation frequency of about $0.5-1 \mathrm{~Hz}$. Interestingly, haemorrhage, in conscious rabbits did not produce any detectable activation of the sympathetic nerves to the uvea.

It seems likely that the role of the sympathetic nerves to the eye is to prevent overperfusion with blood and breakdown of intraocular barriers under conditions of acute widespread sympathetic stimulations and blood pressure rise as in fight or flight situations. Activation of the sympathetic nerve fibres to the eye may give a normal blood flow in the choroid in spite of a rise in blood pressure and vasoconstriction in extraocular parts of the vessels supplying the retina may assist myogenic and metabolic autoregulatory mechanisms in preventing overperfusion in the retina. ${ }^{8}$ One can speculate that localised autonomic neuropathy as in diabetes may be a risk, sympathetic protection being less efficient than normally.
In rabbits sympathetic nerve stimulation results in vasoconstriction even after massive blockade of the adrenergic $\alpha$-receptors, which suggests that there may be a release of a nonadrenergic co-transmitter, (Fig. 1). In other systems neuropeptide $\mathrm{Y}$ plays this role. This neuropeptide is present in the adrenergic

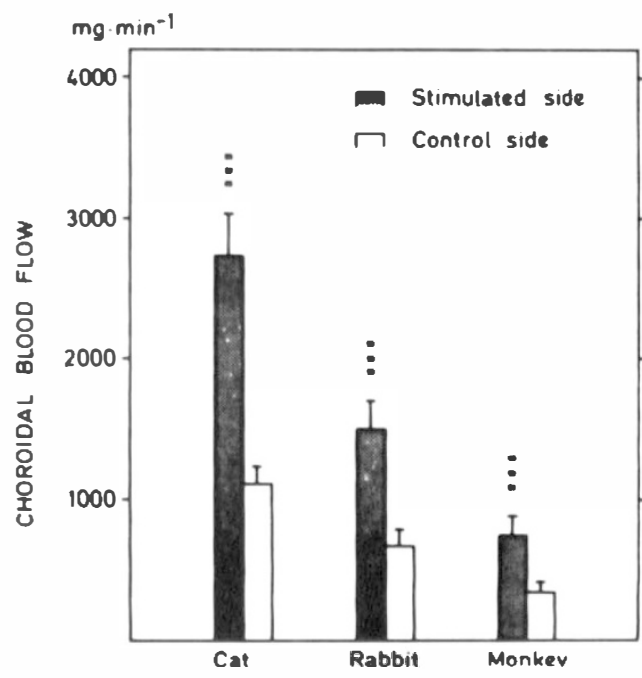

Fig. 2. Near maximal effects of electrical stimulation of the parasympathetic division of the facial nerve on choroidal blood flow in different species. From Nilsson ${ }^{10}$. 
nerves of the choroid and when injected intravenously it produces vasoconstriction. It is thus likely to be the unknown co-transmitter also in the eye. ${ }^{9}$

Electrical stimulation of the facial nerve intracranially or the greater petrosal nerve via the middle ear results in marked vasodilatation in the uvea and increased blood flow, ${ }^{10}$ (Fig. 2). The effect is frequency dependent with near maximal effects at $10 \mathrm{~Hz}$. The effect is nonmuscarinic and therefore due to some transmitter other than acetylcholine. Vasoactive intestinal peptide, VIP, is a good candidate at least in rabbits. The peptide is present in the nerves originating in the pterygopalatine ganglion and it is a potent vasodilator in the choroid. Peptide HI, PHI, is another candidate, possibly released in parallel with VIP. However, in cats none of these peptides is a potent vasodilator. Thus there may be species variations with regard to the transmitter although the effect of nerve stimulation is the same.

The physiological importance of the facial nerve induced vasodilatation in the choroid is not at all clear. It has been reported by Par$\mathrm{ver}^{11}$ that during light exposure of one eye in primates there was a rise in temperature in the fellow eye indicating vasodilation. The afferent as well as efferent fibres involved in the reflex were thought to be carried in the optic nerve. It seems possible, however, that the reflex might involve the facial nerve mechanism. One can speculate also that the facial
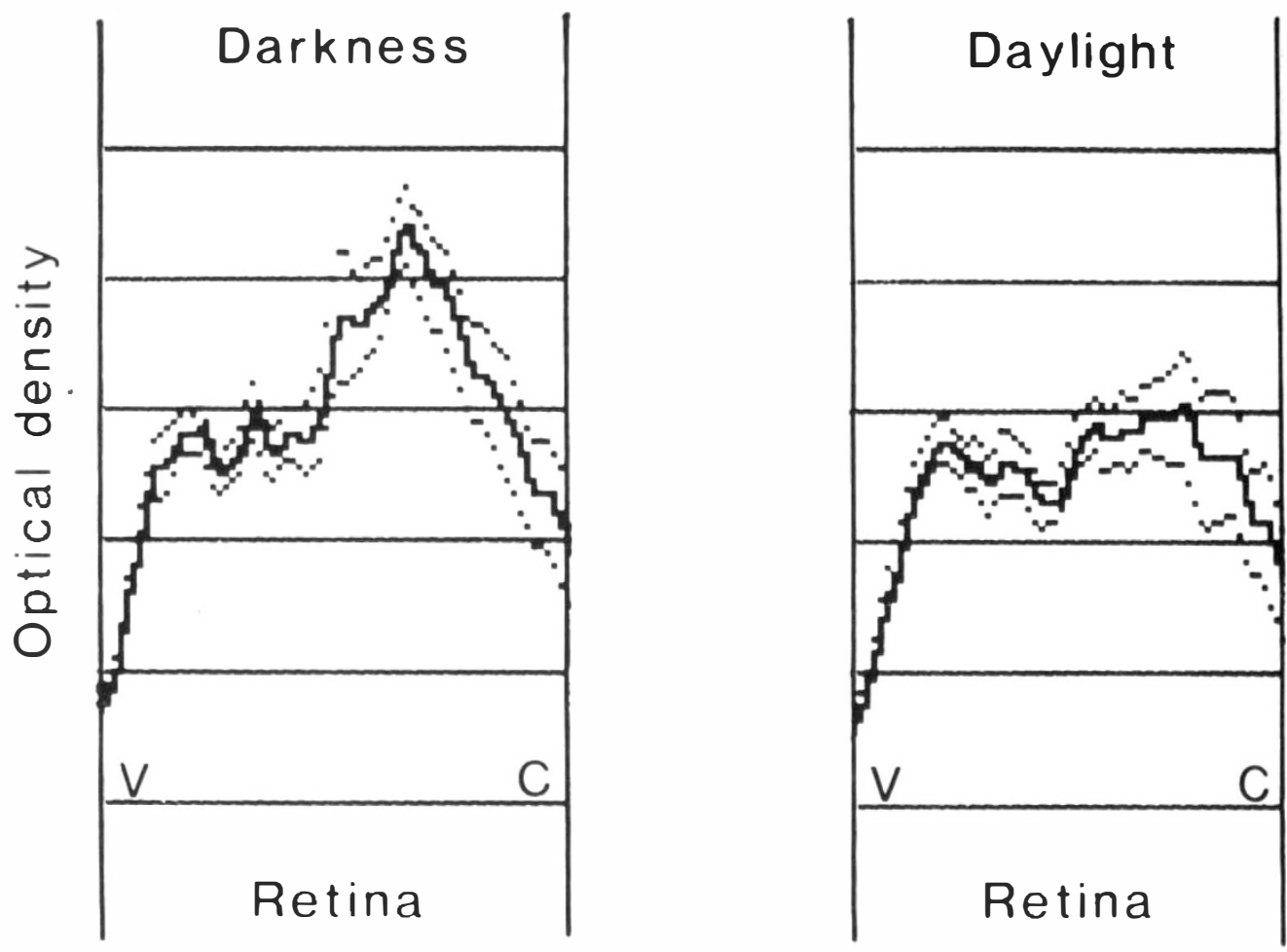

$2 \mathrm{~mm}$. temporal of optic nerve, $M$ and SEM,

12 sections. $\mathrm{V}=$ vitreous, $\mathrm{C}=$ choroid .

Fig. 3. Effect of constant daylight on the accumulation of ${ }^{3} H$-2-deoxyglucose in the retina. The average optical density profile in autoradiographs of the retina at a position $2 \mathrm{~mm}$ temporal of the optic nerve head shows that constant light caused a moderate reduction in accumulation of tracer in the outer part of the retina. 
nerve mediated vasodilatation might be of importance during sleep; there is then a combination of darkness, which stimulates photoreceptor metabolism and episodes with marked reductions in blood pressure. According to a recent report there is a rise in intraocular pressure in the first 30 minutes after subjects sleep. ${ }^{12}$ This rise might be due to parasympathetic activation. Interestingly, the nerves are not activated by a blood pressure fall caused by haemorrhage. ${ }^{13}$

\section{Effects of light on retinal metabolism}

$\mathrm{We}^{14}$ have recently studied the effects of light in cynomolgus monkeys (Macaca fascicularis) on retinal metabolism with the 2-deoxyglucose method developed by Sokoloff ${ }^{15}$ for studies on cerebral metabolism. In the experiments, one eye had the lids sutured and the whole orbit covered with black fabric and plastic. The other was exposed in some experiments to laboratory light or daylight reflected from a white screen placed in front of the animal, in other experiments to flicker-

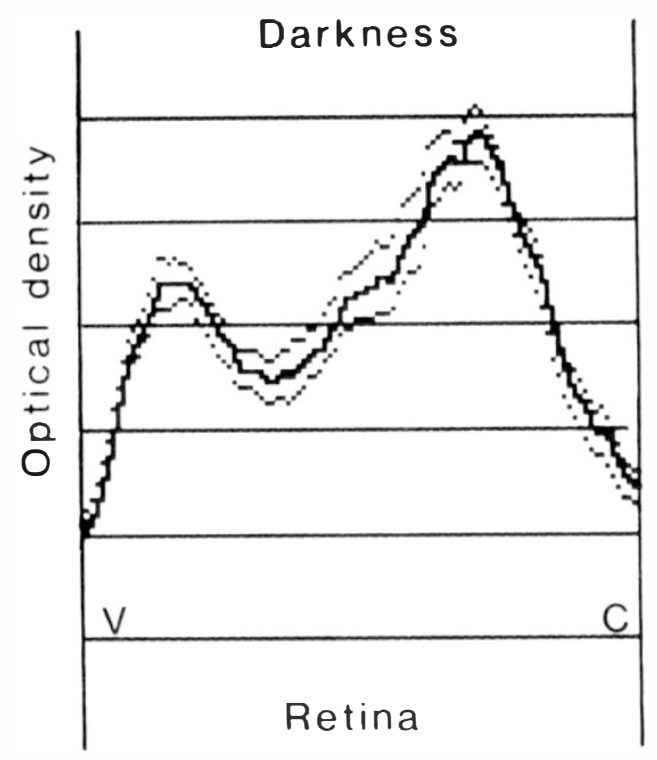

ing light from a stroboscope reflected from the same screen. After dark exposure of the control eye for at least 30 minutes and light exposure of the experimental eye for at least 10 minutes deoxyglucose was injected intravenously and 40 minutes later the animal was killed and paracentesis was performed bilaterally. The whole head was frozen at $-40^{\circ} \mathrm{C}$, sectioned according to Ullberg ${ }^{16}$ and autoradiographs were made.

Figure 3 shows densitometric scans from the retinal side to the choroidal in an experiment with daylight on one side. Such light at about 220 Lux moderately reduced (about $30 \%$ ) the accumulation of tracer in the outer part of the retina but had no clear effect on the inner layers. This reduction indicates a reduction in glucose metabolism. With flickering light at $8 \mathrm{~Hz}$ there was a moderate increase (about $30 \%$ ) in accumulation of tracer in the inner parts of the central retina indicating enhanced glucose consumption but no clear effect in the outer parts, (Fig. 4). These observations are in agreement with observation of

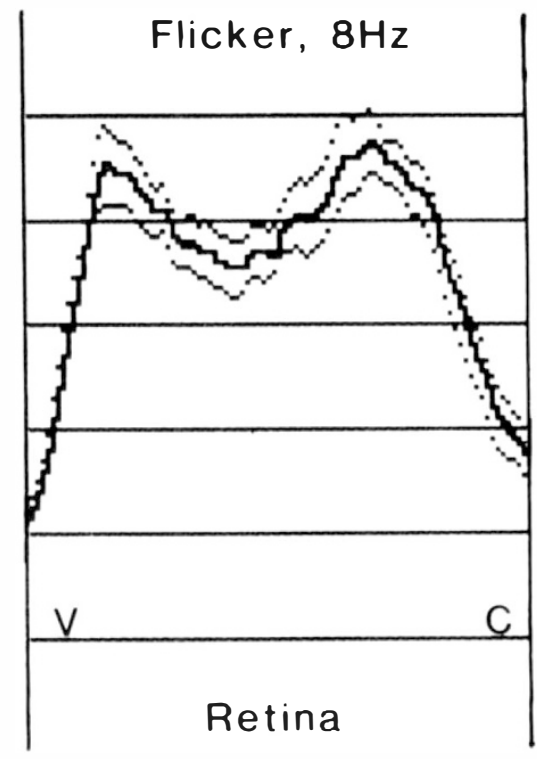

$1 \mathrm{~mm}$. temporal of optic nerve, M and SEM,16 sections.

$\mathbf{V}=$ vitreous, $\mathbf{C}=$ choroid .

Fig. 4. Effect of flickering light on the accumulation of ${ }^{3} \mathrm{H}$-2-deoxyglucose in the retina. The average optical density profile in autoradiographs of the retina at a position $1 \mathrm{~mm}$ temporal of the optic nerve head shows that constant light caused moderate enhancement of tracer accumulation in the inner part of the retina. $V$ : vitreious side, $C h$ : choroidal side of the retina. 
Table I Effects of flickering light, $8 \mathrm{~Hz}(n=8)$, and daylight $(n=10)$ as compared to darkness on retinal and choroidal blood flow. Mean values $\pm S E$.

\begin{tabular}{lcc}
\hline & \multicolumn{2}{c}{ Blood flow, g/min } \\
& Retina & Choroid \\
\hline $8 \mathrm{~Hz}$ & $0.062 \pm 0.0066$ & $1.23 \pm 0.11$ \\
dark & $0.052 \pm 0.0076$ & $1.34 \pm 0.16$ \\
light & $0.046 \pm 0.0040$ & $1.30 \pm 0.19$ \\
dark & $0.057 \pm 0.0052$ & $1.25 \pm 0.17$ \\
\hline
\end{tabular}

Sickel ${ }^{17}$ that in the isolated frog retina constant light reduced the metabolism while the onset of light caused a spurt of metabolism.

\section{Effects of light on retinal and choroidal blood flow}

The labelled microsphere method was used as described elsewhere ${ }^{18}$ to determine the effects of light on retinal and choroidal blood flow rates. The animals were anaesthetised with urethan, arterial blood pressure, oxygen and carbon dioxide tensions and $\mathrm{pH}$ were checked to have normal values and blood flow determinations were made at times which made the results comparable to those performed with the deoxyglucose method. It was found in these experiments, Table I, that retinal blood flow tended to be higher in the eye subjected to flickering light than in the control, the difference being $0.010 \pm 0.004 \mathrm{~g} / \mathrm{min}(\mathrm{n}=8$, $\mathrm{p}=0.03$ with one-tailed t-test). In the eye subjected to constant light the retinal blood flow tended to be lower than in the control, this difference being $0.011 \pm 0.05 \mathrm{~g} / \mathrm{min}$ $(\mathrm{n}=10, \mathrm{p}=0.03)$.

It is of interest also to compare retinal blood flow values for the retina under flickering light and constant light. Under flickering light, the blood flow was $0.016 \mathrm{~g} / \mathrm{min}$ higher than in constant light $(p=0.02)$. These results suggest that the changes in retinal blood flow that were caused by light were small, flickering light tending to increase the blood flow by some $20 \%$ and constant light tending to reduce the flow by a similar percentage.

There was no evidence for a unilateral effect of light on choroidal blood flow. However, the possibility of a bilateral effect of light on choroidal blood flow (cf Parver ${ }^{11}$ ) cannot be ruled out. If that occurred it was similar in experiments with constant light to those with flickering light in spite of the fact that the metabolism in the outer retina changed only in constant light and was then reduced. Thus, if vasodilatation occurred it was not an adjustment to increased metabolic needs of the outer retina. The small effect of light on retinal blood flow observed in the present study is at variance with the results of previous studies, ${ }^{19,20}$ in humans using the laser Doppler velocitometry method; according to these studies retinal blood flow increased by $67 \%$ and $40-70 \%$, respectively, during dark exposure. It seems possible that the laser light stimulated the metabolism of the inner retina resulting in a spurt of oxygen consumption and local vasodilatation.

\section{Metabolism and blood flow}

The results of our recent studies in monkeys are in good agreement with the hypothesis that retinal blood flow is autoregulated, local metabolic factors playing an important role in preventing deficient nutrition. Choroidal blood flow on the other hand is not autoregulated and independent of the metabolism in the outer retina; it seems to be set at a noncritical level in the sense that it is high enough to permit adequate nutrition under physiological variations in metabolism in the outer retina. One can speculate that the role of the choroid in retinal temperature control has determined the set point. ${ }^{21}$ This of course does not mean that the flow is high enough to provide the nutrients necessary under all conditions such as at high altitudes or at abnormal arterial or intraocular pressures. In cats even moderate increments in IOP caused alterations in the dark-adapted standing potential ${ }^{22}$ which may be a sign of hypoxia-induced reduction in metabolism. ${ }^{23}$ Such metabolic adaptation may explain why even marked reductions in perfusion pressure have only moderate effects on the glucose consumption in the retina in monkeys; evidence for enhanced anaerobic glycolysis was found only at perfusion pressures that reduced also the retinal blood flow. ${ }^{24}$

A fall in local oxygen tension in the outer retina in darkness has been reported by Linsenmeier ${ }^{6}$ and was suggested to be due to enhanced metabolism under such conditions. 
The experiments with deoxyglucose and blood flow determinations reported here support this opinion. A high metabolic rate in the photoreceptor layer of the retina can be expected in darkness due to the dark current which is due to enhanced sodium leakage into the cells; rising intracellular sodium concentrations are likely to stimulate the $\mathrm{Na} / \mathrm{K}$ ATPase involved in sodium transport out of the cells. ${ }^{25}$.

\section{Effects of circulating substances}

A number of transmitters and hormones acting from the blood side may be expected to affect the choroidal vessels and there may be effects also on the retinal vessels. ${ }^{1}$ Epinephrine, norepinephrine and angiotensin II may be mentioned here. However, none of these can be expected to be involved in the regulation of retinal and choroidal blood flow according to the varying needs of the retina.

We wish to thank Ms Lena Stjernschantz and Mr Alf Johansson for expert technical assistance.

Supported by Grant 5Ro1 EY 00475 from the National Eye Institute, US Public Health Service and by Grant 88-04X-00147 from the Swedish Medical Research Council.

\section{References}

${ }^{1}$ Bill A: Circulation in the eye. in Renkin EM, Michel CC eds. Handbook in physiology. Microcirculation, Part 2, The American Physiological Society 1984, 1001-34.

${ }^{2}$ Robinson F, Riva EC, Grunwald JE, Petrig BL, Sinclair SH: Retinal blood flow autoregulation in response to an acute increase in blood pressure. Invest Ophthalmol Vis Sci 1986, 27: 722-6.

${ }^{3}$ Törnquist $\mathrm{P}$ and Alm A: Retinal and choroidal contribution to retinal metabolism in vivo. A study in pigs. Acta Physiol Scand 1979, 106: 315-3.

${ }^{4}$ Bill A, Törnquist P, Alm A: Permeability of the intraocular blood vessels. Trans Ophthalmol Soc, UK 1980, 100: 332-6.

${ }^{5}$ Alder WA and Cringle S: The effect of raised intraocular pressure on the intraretinal oxygen profiles. Invest Ophthalmol Vis Sci 1988, 29: 247 (Abstr).

${ }^{6}$ Linsenmeier RA: Effects of light and darkness on oxygen distribution and consumption in the cat retina. J Gen Physiol 1986, 88: 521-42.

${ }^{7}$ Stjernschantz J, Alm A, Bill A: Effects of intracranial oculomotor nerve stimulation on ocular blood flow in rabbits. Modification by indomethacin. Exp Eye Res 1976, 23: 461-70.

${ }^{8}$ Bill A, Linder M, Linder J: The protective role of ocular sympathetic vasomotor nerve in acute arterial hypertension. Bibl Anat 1977, 16: 47-50. (Proceedings of the 9th Europ Conf Microcirculation, Antwerp 1976).
${ }^{9}$ Granstam E and Nilsson SFE: Non-adrenergic sympathetic vasoconstriction in the eye and some other facial tissues in the rabbit. (Submitted for publication) 1989.

${ }^{10}$ Nilsson SFE: Studies on ocular blood flow and aqueous humor dynamics. Acta Universitatis Upsaliensis 1986, 43: 1-38.

${ }^{11}$ Parver LM, Auker CR, Carpenter DO, Doyle T: II Reflexive control in the monkey. Arch Ophthalmol 1982, 100: 1327-30.

${ }^{12}$ Brown B, Wildsoet CF, Swann PG: Sleep, light, dark and intraocular pressure. Invest Ophthalmol Vis Sci Suppl 1989, 30: 28.

${ }^{13}$ Linder J: Effects of facial nerve section and stimulation on cerebral and ocular blood flow in hemorrhagic hypotension. Acta Physiol Scand 1981, 112: $185-3$.

${ }^{14}$ Bill A and Sperber GO: Aspects of oxygen and glucose consumption in the retina; effects of high intraocular pressure and light. Graefe's Arch Ophthalmol (in press).

${ }^{15}$ Sokoloff L: Basic principles in imaging of regional cerebral metabolic rates. In Sokoloff L ed. Brain imaging and brain function. Raven Press, New York, 1985, 1-47.

${ }^{16}$ Ullberg S: The technique of whole body autoradiography. Cryosectioning of large specimens. Science Tools, the LKB Instrument Journal (Bromma, Sweden): special issue on Whole-body Autoradiography, 1977: 1-29.

${ }^{17}$ Sickel W: Retinal metabolism in dark and light. In Handbook of sensory physiology. Fuortes MGF ed. Physiology of Photoreceptor Organs. 1972: 667-26.

${ }^{18} \mathrm{~A} \operatorname{m} \mathrm{A}$ and Bill A: The oxygen supply to the retina. II. Effects of high intraocular pressure on uveal and retinal blood flow in cats. A study with labelled microspheres including flow determinations in brain and some other tissues. Acta Physiol Scand 1972, 84: 306-19.

${ }^{19}$ Feke GT, Zuckermann R, Green GJ, Weiter JJ: Response of human retinal blood flow to light and dark. Invest Ophthalmol Vis Sci 1983, 24: 136-41.

${ }^{20}$ Riva CE, Grunwald JE, Petrig BL: Reactivity of the human retinal circulation to darkness: A laser doppler velocimetry study. Invest Ophthalmol Vis Sci 1983, 24: 737-40.

${ }^{21}$ Bill A, Sperber GO, Ujiie K: Physiology of the choroidal vascular bed. Internat Ophthalmol 1983, 6: 101-7.

${ }^{22}$ Yancey CM and Linsenmeier RA: The electroretinogram and choroidal $\mathrm{PO}_{2}$ in the cat during elevated intraocular pressure. Invest Ophthalmol Vis Sci 1988, 29: 700-7.

${ }^{23}$ Steinberg RH: Monitoring communications between photoreceptors and pigment epithelial cells: effects of "Mild" systemic hypoxia. Invest Ophthalmol Vis Sci 1987, 28: 188-204.

${ }^{24}$ Sperber GO and Bill A: Blood flow and glucose consumption in the optic nerve, retina and brain; effects of high intraocular pressure. Exp Eye Res 1985, 41: 639-53.

${ }^{25}$ Zuckerman R and Weiter JJ: Oxygen transport in the bullfrog retina. Exp Eye Res 1980, 30: 117-7. 